\title{
As Aventuras de Biguió: um jogo educacional sobre coberturas e caminhos de rainhas, torres e cavalos
}

\author{
Douglas Silva de Melo ${ }^{1}$, Fernanda Pires ${ }^{1,2}$, Rosiane de Freitas ${ }^{2}$ \\ ${ }^{1}$ Laboratório de Pesquisa e Desenvolvimento em Tecnologias Educacionais (ThinkTEd) \\ Licenciatura em Computação - Universidade do Estado do Amazonas (UEA) \\ Caixa Postal 69042-270 - Manaus - AM - Brasil \\ ${ }^{2}$ Instituto de Computação (ICOMP) - Universidade Federal do Amazonas (UFAM) \\ Manaus - AM - Brasil \\ \{dsm.lic17,fpires\}@uea.edu.br, rosiane@icomp.ufam.edu.br
}

\begin{abstract}
Resumo. O presente artigo relata o processo de desenvolvimento do jogo educacional "As aventuras de Biguió", um game de aventura estilo dungeon, desenvolvido na game engine Unity3D com a proposta de auxiliar na visualização e abstração de problemas computacionais complexos e no desenvolvimento do Pensamento Computacional (PC). A mecânica o jogo proporciona ao usuário a possibilidade de criar estratégias capazes de solucionar os problemas apresentados na forma de enigmas e puzzles contextualizados de acordo com o storytelling. $O$ aplicativo não possui restrições quanto a faixa etária. Para crianças foi projetado para auxiliar no desenvolvimento do pensamento computacional. Para os estudantes de computação, é possível identificar os problemas computacionais apresentados.
\end{abstract}

\section{Cenário de uso}

Aprender sobre computação vai muito além de saber programar. Trata-se de criar estratégias eficientes para resolução de problemas, otimizar processos, de forma sistematizada. Aplicando conceitos e teoremas matemáticos, pensando nas decisões e caminhos a serem tomados, em como se apropriar dos mais diferentes conhecimentos para criar bons algoritmos.A abstração de um problema é de suma importância nesse processo de pensar em soluções para os cenários apresentados [Raabe and Silva 2005].

As grades curriculares educacionais possuem uma sequência de aprendizagem, que aumenta de complexidade conforme os caminhos percorridos. Nos cursos de computação, com o passar do tempo os problemas computacionais tornam-se mais complexos. Uma vez que as dificuldades iniciais não sejam sanadas, os entraves com relação a compreensão aumentam acarretando em altos índices de reprovação e evasão [Cristovão 2008]. A compreensão dos problemas apresentados auxiliam na definição das estratégias e na tomada de decisões, em caso de ruídos na compreensão, a decisão pode não ser a melhor [Polya 2004].

Os problemas com relação as taxas de reprovação e evasão tem suscitado discussões em torno de abordagens pedagógicas que possam auxiliar nesse problema. Os jogos tem sido cotados como uma alternativa lúdica a ser utilizada na aprendizagem de determinados conteúdos, visto que conseguem captar a atenção dos usuários, proporcionando a visualização, em tempo real, das mais diferentes interações, dependendo direta 
VIII Congresso Brasileiro de Informática na Educação (CBIE 2019)

Anais dos Workshops do VIII Congresso Brasileiro de Informática na Educação (WCBIE 2019)

ou indiretamente do jogador, possibilitando a abstração que seus elementos podem proporcionar [Dresher 2012, da Silva et al. 2014, Tarouco et al. 2014].

Algumas obras na literatura abordam sobre jogos puzzles, mas poucos tratam dos seus algoritmos. Levitin produziu um livro composto de centenas de puzzles, cada um com sua formalização descrita. Esta obra serviu como apoio na compreensão do funcionamento dos problemas inseridos no jogo [Levitin and Levitin 2011]. [Costa 2018] apresenta uma dissertação de mestrado, importante para o presente trabalho, pois nesta obra é feita a relação de complexidade de algoritmos e a avaliação de algoritmos utilizados em jogos, além de demonstrar como um jogo deve apresentar e propor ao jogador a possibilidade de utilizar algoritmos para resolução de problemas.

"As aventuras de Biguió"é um jogo de aventura, em estilo dungeon cujas fases estão distribuídas em puzzles, com gráficos em 2.5D, para plataforma Android. O públicoalvo direto são estudantes de Computação e áreas afins, com o objetivo de proporcionar a visualização de problemas computacionais complexos como o problema da cobertura de vértices, o passeio do cavalo, as 08 rainhas e dos quatro cavalos. O visualização do problema, pode auxiliar na abstração e compreensão do cenário computacional a ser tratado. Para os que não são vinculados a Computação, o jogo pode contribuir com o desenvolvimento do Pensamento Computacional, pois sua mecânica e gameplay apresentam espaços para a resolução de problemas lógicos.

\section{Desenvolvimento}

Para o desenvolvimento do jogo, utilizou-se a Unity versão 2018.2.2f - uma engine que possibilita a criação de jogos 3D - com códigos-fonte em C\# - linguagem de programação orientada a objetos. O jogo é destinado à plataformas mobile (tablets e celulares) com Sistema Operacional Android versão igual ou superior a 4.2. A Figura 1 contém o diagrama do processo de desenvolvimento do jogo, seccionado em etapas.

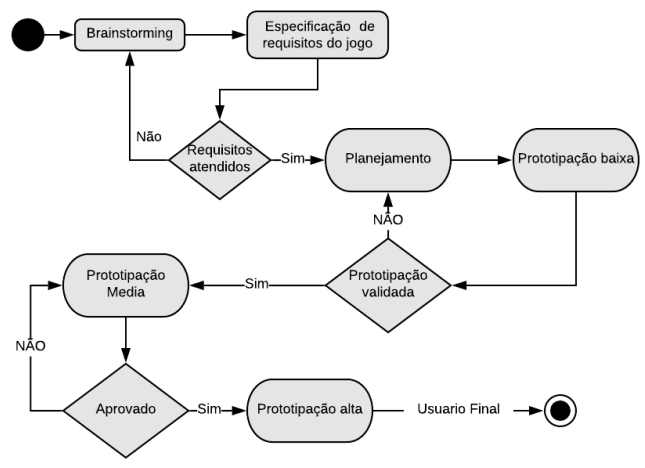

Figura 1. Diagrama de processo de software

Brainstorming: O foco desta etapa foi trocar ideias com os integrantes da equipe para definir tópicos como temática e enredo, baseando-se no problema apresentado, a visualização de problemas computacionais complexos e no público alvo. A partir disso, realizou-se um levantamento na Literatura de trabalhos relacionados com objetivo do jogo para auxiliar na elaboração do aplicativo. Os softwares e trabalhos encontrados que compartilham características comuns foram: 
VIII Congresso Brasileiro de Informática na Educação (CBIE 2019)

Anais dos Workshops do VIII Congresso Brasileiro de Informática na Educação (WCBIE 2019)

Especificação de requisitos de jogos: nessa fase foram definidos os elementos de jogos capazes de gerar flow que poderiam ser considerados na composição, considerando o tema do jogo, o estilo o propósito educacional, a correlação entre ciclo de aprendizagem e sistema de auto regulação de aprendizagem através das fases.

Planejamento: esta etapa ocorreu em dois momentos: $i$ ) definição do cronograma e divisão de atividades e ii) início da documentação do jogo. O modelo de documento utilizado é uma adaptação no Game Design Document (GDD) de Scott Rogers [2014] . O documento conta com a descrição completa do jogo, a jornada do herói alinhada a jornada de conhecimento, bem como a disposição do elementos de aprendizagem de acordo com os cenários do jogo, o storytelling, as mecânicas, a experiência de usuário, etc.

Validação 1: a primeira validação consistiu na análise dos requisitos do jogo e na forma de apresentação destes seguindo a narrativa proposta. Logo, o artefato apresentado nessa etapa é o esquema do jogo e sua concepção segundo os critérios definidos na etapa anterior; caso os requisitos estivessem alinhados, a etapa era validada e o artefato poderia seguir para a criação de protótipo.

Prototipagem Baixa Fidelidade: nessa etapa o artefato apresentado foi o primeiro modelo do jogo em paper prototype, cujo objetivo foi avaliar a jogabilidade e os elementos de usabilidade presentes na interface, bem como a mecânica, storyboard e gameplay.

Validação 2: consistiu na análise do primeiro protótipo digital com o objetivo de verificar se os requisitos de jogo e aprendizagem foram atendidos. Nessa etapa, foram propostas modificações na sequencia de fases e na mecânica do jogo.

Protótipo de média fidelidade: após a ideia do jogo já definida e com a mecânica funcionado no papel, o protótipo de média fidelidade foi desenvolvido. Para esta etapa já foi utilizada a game engine Unity.

Validação 3: a terceira validação teve como objetivo analisar se o jogo estava da forma que foi inicialmente planejado e se suas funcionalidades estavam coesas. Além disso, utilizou-se os testes de usabilidade e percurso cognitivo para validar a experiência de usuário no jogo [Wharton 1994, Nielsen 1993].

Prototipagem de Alta fidelidade: nessa fase, o artefato é o protótipo de alta fidelidade.Todas as correções e alterações necessárias já foram implementadas dando ao jogo uma versão final e, por fim, foi avaliado por um público alvo de dez usuários, foi utilizado os critérios do MEEGA+KIDS de von Wangenheim [2018] .

\section{Apresentação de Software}

"Aventuras de Biguió"é um jogo educacional mobile (android), com visão $2.5 \mathrm{~d}$, estilo Dungeon, temática de jogo em caverna/masmorra com cores escuras, foi desenvolvido com o objetivo de auxiliar no processo de visualização de problemas computacionais. A versão atual do jogo é composta por 4 fases com diferentes mecânicas, que possuem características semelhantes às peças de Xadrez. Em cada fase está presente um problema (puzzle) e para o jogador completá-la, é necessário visualizar e abstrair o problema, para assim formular um algoritmo que possa resolvê-lo. O jogo possibilita que o jogador consiga rastrear seu algoritmo, de modo que cada ação realizada pode ser observada em tempo real, tornando-a lúdica. 
VIII Congresso Brasileiro de Informática na Educação (CBIE 2019)

Anais dos Workshops do VIII Congresso Brasileiro de Informática na Educação (WCBIE 2019)

\subsection{Enredo do jogo}

BiguiÓ (protagonista do jogo) é um jovem explorador que vive na cidade mais evoluída do mundo: Complex City. A cidade possui esse nome devido ao objeto místico conhecido como: "Eficience", que proporciona respostas eficientes para todos os problemas da cidade, como "Qual a melhor forma de distribuir a água com gasto mínimo ?". O objeto é composto por diversos fragmentos que o mantém em perfeito estado. Caso um de seus fragmentos seja desativado, o objeto perde a sua eficiência, não proporcionando mais as melhores soluções. Para ativar um fragmento basta resolver um problema (que se encontra na masmorra).

Certo dia um cidadão exilado desta cidade, em busca de vingança, retirou a solução de todos os puzzles da masmorra que ativam os fragmentos. Agora, a missão de Biguió é resolvê-los novamente para reativar os fragmentos, fazendo com que o objeto funcione corretamente, trazendo paz à cidade.

\subsection{Mecânica do jogo}

"Aventuras de BiguiÓ"utiliza uma mecânica de movimentação semelhante ao das peças do Xadrez, que se movem pelas casas da matriz/tabuleiro. Tendo isto em vista, foram pesquisados jogos com estas funções, dentre estes: Tijox, um jogo que trabalha a ideia de cobertura de vértices; Chesspuzzles, um repositório como diversos problemas de Xadrez que, além de conter diversos problemas, também possui a parte de avaliação dos algoritmos utilizados para resolução dos problemas.

O jogador utiliza o botão analógico para mover o personagem para todas as direções e, por meio do botão de ação, pode interagir com os elementos do jogo. Cada botão e elemento de tela realiza uma função diferente, de acordo com a fase em que está (Figura 2).

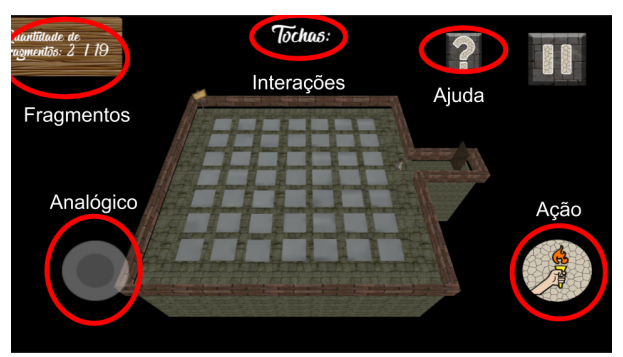

Figura 2. Botões e elementos (Interagir/Placar de fragmentos/ Ajuda/ Analógico/ Placar interações)

Cada fase do jogo possui um problema/desafio e história diferentes, que de forma implícita, auxiliam ao jogador compreender o problema de forma mais lúdica. Por meio de mecânicas únicas em cada fase, o objetivo do jogador é resolver os respectivos problemas, descritos a seguir:

Fase 1 - Ilumine a cidade - Problema da cobertura de Vértice (Figura 3): Em um tabuleiro 7x7, o jogador têm a missão de iluminar (colorir) todo o tabuleiro utilizando tochas. Cada tocha ilumina a posição em que foi colocada, uma casa nas direções verticais e horizontais (formando um sinal de adição "+"), não podendo ser colocada onde houver outra. O objetivo geral é iluminar todas as casas com a menor quantidade de tochas. 
VIII Congresso Brasileiro de Informática na Educação (CBIE 2019)

Anais dos Workshops do VIII Congresso Brasileiro de Informática na Educação (WCBIE 2019)
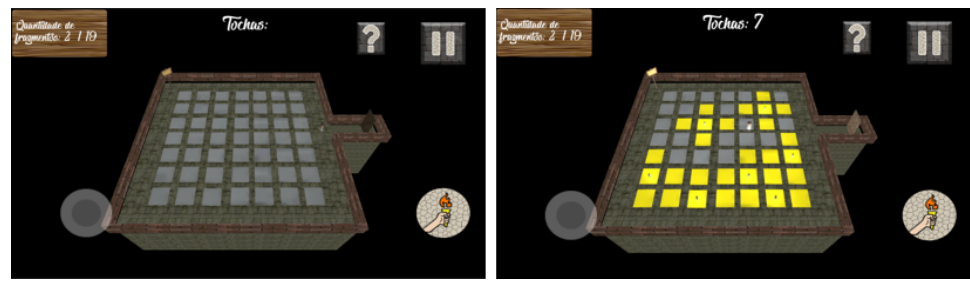

Figura 3. Fase 1 - Estado inicial/Movimento

Fase 2 - Organize meus arqueiros - Problema das 8 rainhas (Figura 4): Posto sobre um tabuleiro $8 \times 8$, o jogador deve organizar oito arqueiros de forma que nenhum entre na área de ataque do outro. Os arqueiros atacam nas mesmas direções que a rainha no xadrez (todas as casas nas diagonais, vertical e horizontal).
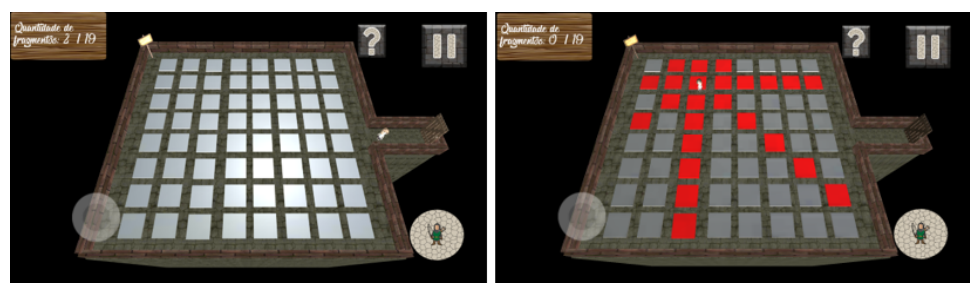

Figura 4. Fase 2 - Estado inicial/Movimento

Fase 3 - Cobrar impostos - Problema do passeio do cavalo (Figura 5): Sobre um tabuleiro 5x5, o jogador deve guiar um cavalo e cobrar impostos de todas as casas do tabuleiro (vilas). O cavalo pode ser movido em L (igual no xadrez) e não é permitido cobrar uma vila mais de uma vez.
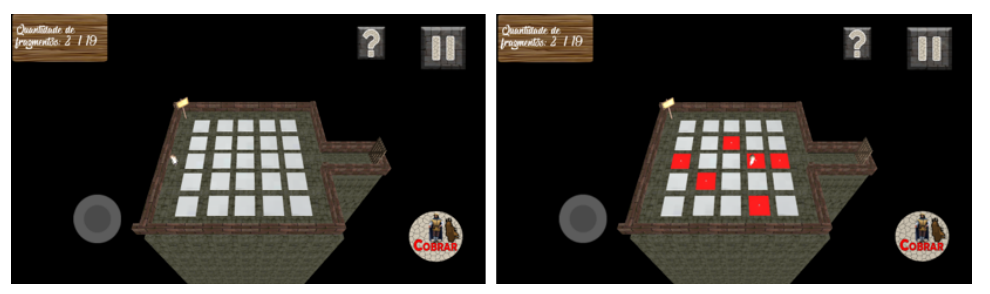

Figura 5. Fase 3 - Estado inicial/Movimento

Fase 4 - Os 4 cavalos guardiões - Problema dos 4 cavalos (Figura 6): Em um tabuleiro 3x3, há quatro cavalos (semelhantes ao do xadrez), dois brancos e dois vermelhos, que estão alocados nas extremidades da matriz. O objetivo do jogador é trocar a posição dos cavalos brancos com a dos vermelhos e vice-versa.
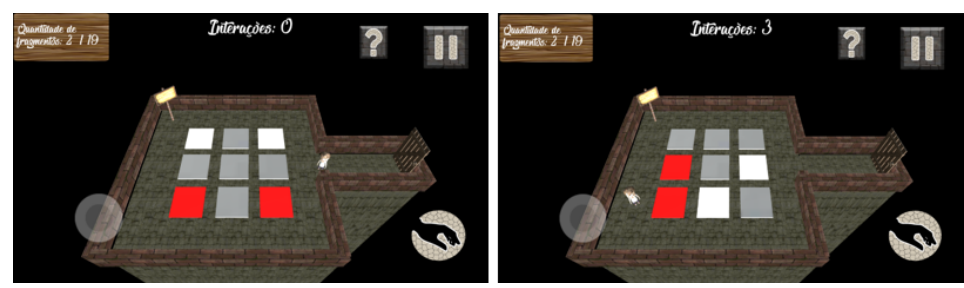

Figura 6. Fase 3 - Estado inicial/Movimento 
VIII Congresso Brasileiro de Informática na Educação (CBIE 2019)

Anais dos Workshops do VIII Congresso Brasileiro de Informática na Educação (WCBIE 2019)

Após o jogador resolver um problema, alguns fragmentos são ativados. Quanto maior a eficiência da resolução do problema, maior a quantidade de fragmentos. Para que o objeto místico da cidade volte a funcionar normalmente, é necessário a ativação de 19 fragmentos.

\subsection{Aprendizagem em jogo}

No jogo "Aventuras de BiguiÓ"a aprendizagem acontece através da exploração.Método que pelo qual o jogador não precisa de um mentor, sendo auto suficiente no processo de aprendizagem, construindo o seu próprio conhecimento, através da interação com a ferramenta, adequando-se aos princípios da Teoria de aprendizagem construcionista de Seymour Papert [1994] . O jogo possui elementos que possibilitam que isto ocorra, como a livre navegação do usuário pelas fases (cada fase é representada por uma porta dentro de jogo e não é necessária condição para que elas sejam acessadas (Figura 13). O jogador aprende a cada vez que interage com objeto, explorando a interface, testando diversas maneiras de se resolver o problema, de forma que não é penalizado por tais ações.

O jogador poderá visualizar sua progressão no jogo, entendendo que está evoluindo; para isso, alguns feedbacks visuais são postos em tela, como por exemplo na Figura 7:

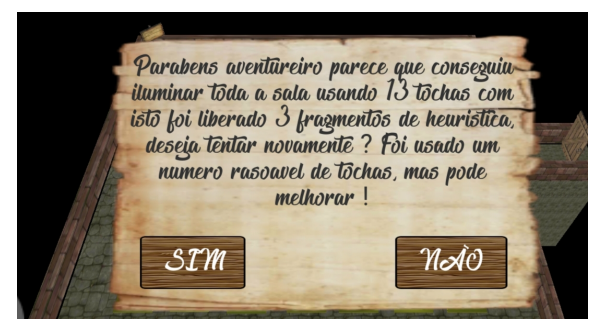

Figura 7. Feedback para avaliação da resolução utilizada no problema

Dependendo do público que está jogando "Aventuras de BiguiÓ”, ele pode ter um foco diferente; para alunos de Ciência da Computação e áreas afins, o jogo tem como objetivo proporcionar a identificação do problema e visualização da ação de determinado algoritmo sobre o desafio escolhido, promovendo um rastreamento do código. Além disto, o jogador pode observar a eficácia do algoritmo utilizado, começando a entender qual algoritmo ele deve optar em determinado caso - tudo isso ocorrendo de forma lúdica. O público geral, ao interagir com o jogo, associa a um game de estratégia e raciocínio lógico.

O jogo possibilita que o jogador utilize - se desejar - algoritmos que já existem na Literatura e que podem ser aplicados nos desafios do jogo; como por exemplo, o problema da Fase 2, que está relacionado com o famoso "Problema das Oito Rainhas". Dessa forma, ele pode tentar entender o algoritmo utilizando o jogo como um rastreador visual, de tal modo que compreenda a ação do algoritmo sobre o problema. O jogo apresenta um storytelling (Figura 8) e tutorial (Figura 9) diferentes para cada um dos problemas apresentados, possibilitando que o jogador compreenda e resolva o problema, melhorando suas habilidades. 


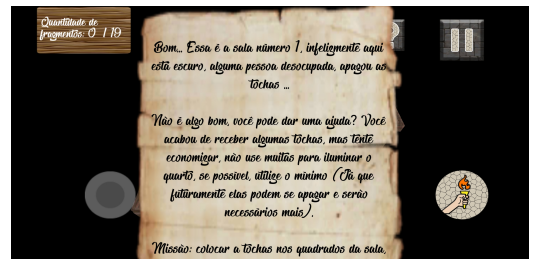

Figura 8. Storytelling especifico que pode ser acessado em cada fase

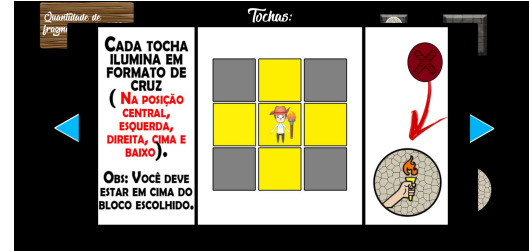

Figura 9. Tutorial de como funciona a mecânica da fase atual

\subsection{Descrição das telas}

A primeira tela que o jogador acessa é o "Menu Principal"(Figura 10), na qual pode ajustar as configurações de som (efeitos sonoros e música) e acessar as telas de "Informações"(Figura 11) e "Storyboard", através do botão de informações e do botão de iniciar, respectivamente.

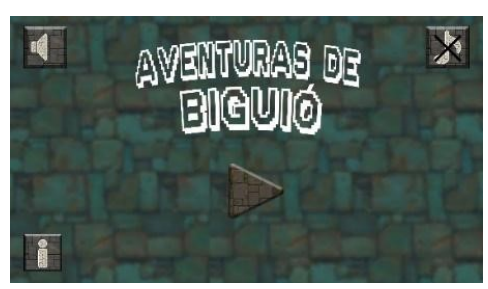

Figura 10. Tela de Menu

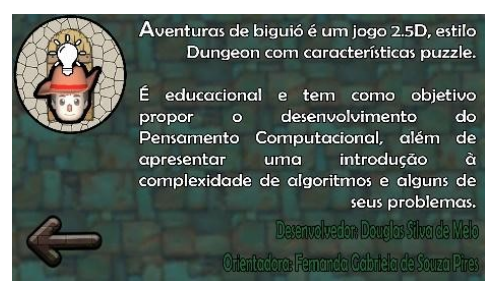

Figura 11. Tela de Informações

Na tela de Storyboard (Figura 12), o jogador visualiza a história do jogo através de diálogos entre os personagens; em seguida, é direcionado para a tela de Fases (Figura 13). A tela de Fases apresenta 4 portas, que correspondem às fases do jogo, com seus desafios específicos (descritos na Seção 3.2). Para acessá-las, basta o jogador movimentar o personagem até próximo à porta e apertar o botão de ação "abrir".

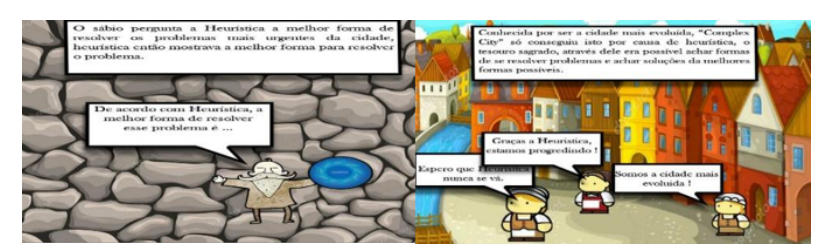

Figura 12. Tela de storytelling

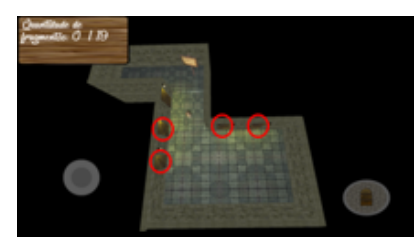

Figura 13. Tela de seleção de fases 
VIII Congresso Brasileiro de Informática na Educação (CBIE 2019)

Anais dos Workshops do VIII Congresso Brasileiro de Informática na Educação (WCBIE 2019)

\section{Considerações finais}

A partir da última versão do jogo "Aventuras de Biguió", o teste MEEGA+KIDS foi aplicado, para analisar critérios de design, experiência de usuário e aprendizagem, sendo as notas atribuídas de 0 a 4 . O resultado obtido foi satisfatório, com uma média 3, demonstrando que o jogo está no caminho certo, mas ainda precisa ser melhorado para futuras publicações. Como pontos de melhoria, pretende-se aprimorar o design de arte do jogo, acrescentar mais fases e aplicar mais testes.

\section{Referências}

Costa, D. M. (2018). Computational complexity of games and puzzles. arXiv preprint arXiv:1807.04724.

Cristovão, H. M. (2008). Aprendizagem de algoritmos num contexto significativo e motivador: um relato de experiência. $S B C, 30$.

da Silva, B. C., Silva, P. P., da Luz, L. P., Silva, E. G., and Martins, H. P. (2014). Jogos digitais educacionais como instrumento didático no processo de ensino-aprendizagem das operações básicas de matemática. In Brazilian Symposium on Computers in Education (Simpósio Brasileiro de Informática na Educação-SBIE), volume 25, page 682.

Dresher, M. (2012). The mathematics of games of strategy. Courier Corporation.

Levitin, A. and Levitin, M. (2011). Algorithmic puzzles. OUP USA.

Nielsen, J. (1993). Response times: the three important limits. Usability Engineering.

Papert, S. (1994). A máquina das crianças. Porto Alegre: Artmed.

Polya, G. (2004). How to solve it: A new aspect of mathematical method. Number 246. Princeton university press.

Raabe, A. L. A. and Silva, J. d. (2005). Um ambiente para atendimento as dificuldades de aprendizagem de algoritmos. In XIII Workshop de Educação em Computação (WEI'2005). São Leopoldo, RS, Brasil.

Rogers, S. (2014). Level Up! The guide to great video game design. John Wiley \& Sons.

Tarouco, L. M. R., Costa, V. M. d., Avila, B. G., Bez, M. R., and Santos, E. F. d. (2014). Objetos de aprendizagem: teoria e prática.

von Wangenheim, C. G., Petri, G., and Borgatto, A. F. (2018). Meega+ kids: A model for the evalua-tion of educational games for compu-ting education in secondary school.

Wharton, C. (1994). The cognitive walkthrough method: A practitioner's guide. Usability inspection methods. 